\title{
Evaluation of a regional real-time precise positioning system in Australia based on GPS/BeiDou observations
}

\author{
Wenwu Dinga,*, Bingfeng Tan ${ }^{a}$, Yongchang Chen ${ }^{a, b}$, Felix Norman Teferlec, Yunbin Yuan ${ }^{a}$ \\ a State Key Laboratory of Geodesy and Earth's Dynamics, Institute of Geodesy and Geophysics, 430077 Wuhan, \\ China \\ b University of Chinese Academy of Sciences, 100049 Beijing, China \\ cInstitute of Geodesy and Geophysics, University of Luxembourg, 4365 Esch-sur-Alzette, Luxembourg
}

\begin{abstract}
The performance of real-time (RT) precise positioning can be improved by utilizing observations from multiple Global Navigation Satellite Systems (GNSS) instead of one particular system. Since the end of 2012, BeiDou, independently established by China, began to provide operational services for users in the Asia-Pacific regions. In this study, an regional RT precise positioning system in Australia is developed to evaluate the performance of GPS/BeiDou observations in providing high precision positioning services for users. Fixing three hourly updated satellite orbits, RT correction messages are generated and broadcasted by processing RT observation/navigation data streams from AUSCORS at the server side. At the user side, RT PPP is realized by processing RT data streams and the RT correction messages received. RT clock offsets, for which the accuracy reached 0.07 and 0.25 ns for GPS and BeiDou, respectively, can be determined. Based on these corrections, an accuracy of 12.2, 30.0 and $45.6 \mathrm{~cm}$ in the North, East and Up directions was achieved for the BeiDou-only solution after 30 minutes while the GPS-only solution reached $5.1,15.3$ and $15.5 \mathrm{~cm}$ for the same components at the same time. A further improvement of 43.7, 36.9 and 45.0 percent in the three directions, respectively, was achieved for the combined GPS/BeiDou solution. After the initialization process, the North, East and Up positioning accuracies were 5.2, 8.1 and $17.8 \mathrm{~cm}$, respectively, for the BeiDou-only solution, while 1.5, 3.0, and $4.7 \mathrm{~cm}$ for the GPS-only solution. However, we only noticed a $20.9 \%$ improvement in the East direction was obtained for the GPS/BeiDou solution, while no improvements in the other directions were detected. It is expected that such improvements may become bigger with the increasing accuracy of the BeiDou-only solution.
\end{abstract}

\section{Keywords}

GPS; BeiDou; precise point positioning (PPP); real-time (RT); satellite clock offset

\footnotetext{
${ }^{*}$ Corresponding author.

E-mail address: dingwenwu@asch.whigg.ac.cn(Wenwu Ding), bingfengtan@asch.whigg.ac.cn(Bingfeng Tan), chenyongchang@whigg.ac.cn(Yongchang Chen), Norman.Teferle@uni.lu(Felix Norman Teferle), yybgps@asch.whigg.ac.cn(Yunbin Yuan)
} 


\section{Introduction}

Real-time (RT) attracts much attention from scientific researchers and companies involved in the development of Global Navigation Satellite Systems (GNSS) for various applications, e.g. precise positioning, timing, and meteorology (de Haan et al., 2009; Defraigne et al., 2015; Geng et al., 2011). In addition, precise point positioning (PPP) has become very popular because of its accuracy and flexibility compared with the classic network solution (Zumberge et al., 1997). However, the performance of the PPP processing strategy is more dependent on the accuracy of the satellite products, especially the satellite clock offsets. Moreover, the accuracy of satellite clock offsets decreases rapidly when predicted (Douša, 2010; Lou et al., 2014), as is needed for RT applications. Hence, the broadcast ephemeris and the predicted part of ultra-rapid products provided by the International GNSS Service (IGS) (Dow et al., 2009), which can both be obtained in RT, cannot satisfy the accuracy requirements in RT PPP (Elsobeiey and Al-Harbi, 2016). To solve the problem, extensive researches have been conducted in recent years. The IGS RT Service (RTS), which was launched in 2013 following the completion of the IGS RT Pilot Project, can provide RT satellite orbit/clock products for public users (Caissy et al., 2012). Analysis results reveal that the accuracy of IGS RT orbit/clock products is $5 / 8 \mathrm{~cm}$ for GPS and $13 / 24 \mathrm{~cm}$ for GLONASS by comparing with European Space Agency (ESA)/European Space Operations Centre (ESOC) final products (Hadas and Bosy, 2015). Based on the products, positioning results with an average accuracy of 16, 9 and $23 \mathrm{~cm}$ for the East, North and Up components in kinematic PPP can be achieved (Elsobeiey and Al-Harbi, 2016). In addition, various institutes also provide their RT products independently, and some companies provide positioning services for commercial users (Leandro et al., 2011; Tegedor et al., 2015). All these developments enable RT PPP to be widely used in recent years.

One drawback of RT PPP exists in the relatively long initialization time and low accuracy. To overcome this deficiency, it is a good choice to realize RT PPP incorporating observations from multiple GNSS. Analysis results based on GPS/GLONASS observations already validated this idea (Cai and Gao, 2013). Currently, additionally to GPS and GLONASS, China is developing its own navigation satellite system - BeiDou. The first phase of this system was to provide an operational service over the Asia-Pacific regions and this was completed by the end of 2012. The ongoing second phase is to establish a system consisting 5 Geostationary Earth Orbit (GEO), 3 Inclined Geo-Synchronous Orbit (IGSO) and 27 Medium Earth Orbit (MEO) satellites, and is scheduled to be completed in 2020 (Sun et al. 2012). During the period of this study, there were 5 GEO, 6 IGSO and 3 MEO satellites in orbit. Various studies on precise applications based on BeiDou or GNSS observations have been conducted in recent years, and comparative performance can be obtained based on BeiDou-only observations compared with the existing GNSS (Li et al., 2014; Montenbruck et al., 2013; Steigenberger et al., 2013; Tegedor et al., 2014). In RT mode, the simulated analysis of RT PPP based on GNSS observation files also revealed that the accuracy of RT BeiDou PPP is comparable to that of RT GPS PPP (Li et al., 2015; Zhang et al., 2016). Since November 2015, the Centre National d'Etudes Spatiales (CNES) began to provide RT BeiDou orbit/clock corrections to public users via the mountpoint CLK93 (Laurichesse, 2015). Fugro realized RT generation of orbit/clock products for BeiDou based on the observations from the IGS Multi-GNSS Experiment (MGEX) (Montenbruck et al., 2017) and their proprietary station network, 
and evaluated the performance on a global scale (Tegedor et al., 2015). The accuracy of BeiDou PPP utilizing these products can be better than $1 \mathrm{dm}$ in horizontal and $2 \mathrm{dm}$ in vertical directions, and BeiDou can improve the positioning accuracy and reduce the convergence time by combining it with GPS. Their system, utilizing observations of four GNSS (GPS, GLONASS, BeiDou and Galileo) and being commercialized as G4, has been introduced (Tegedor et al., 2015). All these results suggest that the performance in RT PPP can be further improved by incorporating GPS/BeiDou observations. Lou et al. (2014) pointed out that the impact of orbit errors on RT PPP varied with the size of the reference network since these orbit inaccuracies will be partially absorbed by the clock offsets. Considering that BeiDou is currently providing service for Asia-Pacific users and the orbit accuracy of BeiDou satellites, especially that of GEO, is relatively lower compared with GPS, it is meaningful to conduct an experiment to evaluate the positioning performance at the regional scale, of which the results will provide a valuable reference for establishing the regional precise positioning system based on GPS/BeiDou observations on a regional or continental scale, such as China and Australia.

In this study a RT precise positioning system utilizing GPS/BeiDou observations is realized and an experiment based on the actual RT streams in Australia is conducted to analyze the actual performance which can be achieved. In section 2, we introduce the method of RT clock offset estimation and PPP, as well as discuss relevant settings during our data processing. The tracking network and organization of the experiment are introduced in section 3. Analysis results based on GPS/BeiDou observations are shown and discussed in detail in section 4. Some conclusions are given in section 5 .

\section{Data Processing Strategy}

\subsection{RT clock offset estimation method}

In RT satellite clock offset estimation, the speed of data processing can be accelerated by epoch differencing (Ge et al., 2012). In this research, we adopt the method and extend it to process GPS/BeiDou observations together. We firstly apply epoch differencing to the ionosphere-free phase observations to eliminate ambiguities and ignore the short-term variation of the receiver inter-system bias (ISB). The observation equation can be expressed as follows:

$$
L\left(t_{i-1}, t_{i}\right)=\rho\left(t_{i-1}, t_{i}\right)+c \Delta t\left(t_{i-1}, t_{i}\right)-c \Delta T\left(t_{i-1}, t_{i}\right)+m\left(t_{i-1}, t_{i}\right) \Gamma\left(t_{i}\right)+\varepsilon\left(t_{i-1}, t_{i}\right)
$$

$L$ denotes the ionosphere-free phase observation, $t_{i-1}$ and $t_{i}$ represent the previous and current epoch respectively, $\rho$ represents the geometric distance between satellite and receiver, $\Delta t$ denotes the receiver clock offset, $\Delta T$ represents the satellite clock offset, $\Gamma$ denotes the zenith non-hydrostatic (wet) delay (ZWD), while the hydrostatic delay is corrected by the a-priori model, $m$ denotes the mapping function of ZWD, $c$ is the speed of light, $\varepsilon$ represents the sum of multipath and random noise of phase observation.

We select one reference station and fix the receiver clock variation to the approximate results from standard point positioning (SPP) based on the broadcast ephemeris. For all other stations, we apply satellite differencing again to eliminate the receiver clock offsets and the observation equation becomes:

$$
L^{p q}\left(t_{i-1}, t_{i}\right)=\rho^{p q}\left(t_{i-1}, t_{i}\right)-c \Delta T^{p q}\left(t_{i-1}, t_{i}\right)+m^{p q}\left(t_{i-1}, t_{i}\right) \Gamma\left(t_{i}\right)+\varepsilon^{p q}\left(t_{i-1}, t_{i}\right)
$$


$p, q$ are the satellites. Combining equation (1) and (2), we can estimate the satellite clock variation between epochs and ZWD.

Secondly, we import the results of clock offset variation and ZWD generated to estimate the initial satellite clock offsets at the first epoch based on code observations. The observation equation can be expressed as follows:

$$
P\left(t_{i}\right)=\rho\left(t_{i}\right)+c \Delta t\left(t_{i}\right)-c\left(\Delta T\left(t_{0}\right)+\sum_{j=1}^{i} \Delta T\left(t_{j-1}, t_{j}\right)\right)+m\left(t_{i}\right) \Gamma\left(t_{i}\right)+c I S B\left(t_{i}\right)+\sigma\left(t_{i}\right)
$$

$P$ denotes the ionosphere-free code observation, ISB represents the receiver ISB and is zero for GPS observation, $\sigma$ denotes the sum of multipath and random noise of code observation.

The receiver clock offset of the reference station is fixed to the SPP results as well. For all other stations, we also apply satellite differencing to eliminate the receiver clock offset and the observation equation can be written as follows:

$$
P^{p q}\left(t_{i}\right)=\rho^{p q}\left(t_{i}\right)-c\left(\Delta T^{p q}\left(t_{0}\right)+\sum_{j=1}^{i} \Delta T^{p q}\left(t_{j-1}, t_{j}\right)\right)+m^{p q}\left(t_{i}\right) \Gamma\left(t_{i}\right)+c \delta I S B\left(t_{i}\right)+\sigma^{p q}\left(t_{i}\right)
$$

$\delta$ equals to 0 when the two satellites belong to the same satellite system, and 1 otherwise. In this step, only the satellite clock offsets at the first epoch and ISB parameters are estimated.

Combining the results, the final clock offsets can be generated and expressed as follows:

$$
\Delta T\left(t_{i}\right)=\Delta T\left(t_{0}\right)+\sum_{j=1}^{i} \Delta T\left(t_{j-1}, t_{j}\right)
$$

\subsection{RT kinematic PPP method}

In RT kinematic PPP, ionosphere-free code and phase observations are processed. Station coordinates, receiver clock offset, ZWD and float ambiguities are estimated as unknowns. In addition, one receiver ISB is also estimated as constant when processing GPS/BeiDou observations together. No temporal correlations of station coordinates are considered in kinematic mode.

In addition, the Helmert variance component estimation method is applied in RT GPS/BeiDou PPP to adjust the weights between the two systems (Cai et al., 2014), and the minimum ratio between variances of BeiDou and GPS observations is set to 4 by considering the accuracies of satellite products. The basic formula of the variance component estimation method can be written as follows:

$$
\left[\begin{array}{cc}
n_{1}-2 \operatorname{tr}\left(N_{1} N^{-1}\right)+\operatorname{tr}\left(N_{1} N^{-1} N_{1} N^{-1}\right) & \operatorname{tr}\left(N_{1} N^{-1} N_{2} N^{-1}\right) \\
\operatorname{tr}\left(N_{1} N^{-1} N_{2} N^{-1}\right) & n_{2}-2 \operatorname{tr}\left(N_{2} N^{-1}\right)+\operatorname{tr}\left(N_{2} N^{-1} N_{2} N^{-1}\right)
\end{array}\right]\left[\begin{array}{c}
\hat{\sigma}_{01}^{2} \\
\hat{\sigma}_{02}^{2}
\end{array}\right]=\left[\begin{array}{c}
V_{1}^{T} P_{1} V_{1} \\
V_{2}^{T} P_{2} V_{2}
\end{array}\right]
$$

$n_{i}, N_{i}(i=1,2)$ are the number of observations and the normal matrix for GPS and BeiDou, respectively. $N$ is the sum of normal matrix, $\hat{\sigma}_{0 i}^{2}(i=1,2)$ are the post-processing variance of GPS and BeiDou, $V_{i}(i=1,2)$ are the observation residuals after parameter estimation, $P_{i}(i=1,2)$ are the weight matrices generated based on the a-priori variance for GPS/BeiDou. 
The process is iterated until $\hat{\sigma}_{02}^{2} / \hat{\sigma}_{01}^{2}=1$.

\subsection{Settings in the data processing}

In RT clock offset estimation and the following kinematic PPP, observations of L1, L2 frequencies for GPS and B1, B2 frequencies for BeiDou are applied. The satellite P1-C1 biases are corrected by the differential code bias (DCB) products provided by Center for Orbit Determination in Europe (CODE) (Dach et al. 2009), and the receiver part is absorbed by the receiver clock offset. Wanninger and Beer (2015) analyzed the elevation-dependent biases of BeiDou code measurements and determined the frequency-dependent corrections for MEO and IGSO satellites. In this analysis, we applied the corrections based on the values provided in their paper. For data quality control, the outliers and cycle slips were initially detected by a series of observation combinations in both procedures, including the Melbourne Wübbena (MW), geometry-free and ionosphere-free combination. Observation residuals after parameter estimation were then analyzed to further detect the outliers in RT clock offset estimation, while the Detection, Identification and Adaptation (DIA) method was applied in RT PPP (Tiberius, 1998).

The phase center offset (PCO) and phase center variation (PCV) correction values for GPS and BeiDou satellites were obtained from the latest igs08.atx antenna calibration file, except for BeiDou IGSO and MEO satellites where the information provided by Guo et al (2016) was adopted. The receiver PCO and PCV calibration values from the igs08.atx file were also applied to GPS, and the same receiver PCO corrections as GPS were applied to BeiDou, which may introduce an additional uncertainty at centimeter level (Tegedor et al., 2014). No receiver PCV corrections for BeiDou were applied. All other settings utilized in RT clock offset estimation and PPP are listed in Table 1.

\section{Design of RT system}

\subsection{Tracking network}

The national network of GNSS Continuously Operating Reference Stations (CORS) in Australia was densified to establish a world class infrastructure for earth and geospatial sciences (Janssen, 2009). Since the first AUSCORS station was established in September 2008, the number of reference stations increased steadily, and more GNSS stations were deployed in recent years to meet development needs. Other than the observation files, users can also access the RT data stream via the AUSCORS Ntrip Broadcaster, which provides a good opportunity for this analysis.

In the experiment, we select 51 stations, of which the distribution is plotted in Fig. 1. All these stations can track GPS/BeiDou satellites. Among them, 33 stations (denoted as blue circles) are applied as reference stations for clock offset estimation, while the other 18 stations (denoted as red circles) are applied as user stations for RT kinematic PPP to evaluate the performance.

\subsection{Structure of RT system}

We established a regional RT precise positioning system at the Institute of Geodesy and Geophysics (IGG) of the Chinese Academy of Sciences (CAS) to conduct the analysis. The structure of the RT system is shown in Fig. 2. GNSS observation files from IGS and MGEX were processed to determine the satellite orbit every 3 hours in a batch data processing mode. Considering the time consumed in the data processing, the interval from 1 to 4 hours of the predicted half was 
normally applied and fixed in the clock offset estimation. In addition, the actual satellite position of BeiDou satellites was calculated from a weighted average of the results calculated from previous and current satellite ephemeris. This eliminated the relatively big discontinuities. The equation can be expressed as follows:

$$
\text { pos }=(1-w) \operatorname{pos}_{1}+\text { ppos }_{2}, \quad w=\Delta t / l e n
$$

in which, pos represents the weighted average satellite position, $\operatorname{pos}_{i}(i=1,2)$ denotes the satellite position calculated from previous and current ephemeris, $w$ denotes the weighting factor, $\Delta t$ represents the length of interval from the beginning time of current ephemeris to current epoch, len denotes the update interval of the ephemeris, and equals to 3 hours in this analysis.

In the clock offset estimation process, RT navigation/observation streams from AUSCORS were received in RTCM standard and then provided via shared memory to the RT clock offset estimation software, in which satellite clock offsets were calculated every 3 seconds. Following on, satellite orbit, low-rate and high-rate clock correction messages, which were updated every 3 seconds, 3 minutes and 3 seconds each, are generated and broadcasted to the users via internet.

At the user side, RT navigation/observation streams were received along with the orbit/clock correction messages, and then provided via shared memory to the RT PPP software, in which precise user positions were determined.

\section{Results and Discussions}

The experiment conducted lasted for four days (February 14-17, 2017). The satellite clock offsets were generated in true RT mode. At the user side, the orbit/clock correction messages were collected together with the data streams, and then reprocessed in a replay mode. Three solutions (GPS-only, BeiDou-only and GPS/BeiDou) were generated and compared to analyze the performance of RT kinematic PPP based on single/multi-system observations.

\subsection{Accuracy of orbit/clock results}

The accuracy of satellite orbits utilized in this analysis was evaluated by comparing with the final GNSS orbits calculated at the IGG supported by the International GNSS Monitoring and Assessment (iGMAS). The accuracy of the final GNSS orbits reached $2.1 \mathrm{~cm}$ for GPS by comparing with the IGS final products, while 22.7 (GEO) and 9.9 (IGSO/MEO) $\mathrm{cm}$ for BeiDou compared to the iGMAS final products (Yuan et al., 2016). The mean RMS of each satellite during the period was summarized separately and is shown in Fig. 3 . In addition, the mean results of different satellite types were given in Table 2.

The RMS between GPS satellites was similar and smaller than for BeiDou. The RMS in the radial direction was smaller than $4 \mathrm{~cm}$ for all satellites. The accuracy in the along-track direction was relatively lower than for the other two directions, but still better than $10 \mathrm{~cm}$ except for two satellites. The mean RMS in the radial, along-track and cross-track directions is 2.8, 7.7 and 5.5 $\mathrm{cm}$, respectively. For the BeiDou IGSO satellites, the RMS in the radial direction was smaller than $40 \mathrm{~cm}$, while it was smaller than $2 \mathrm{~m}$ in the along-track and cross-track directions. The mean values for the 6 IGSO satellites were 29.5, 158.1 and $113.5 \mathrm{~cm}$ for the radial, along-track and 
cross-track directions, respectively. The accuracy of the three MEO satellites was better than those of the IGSO satellites in the radial and cross-track directions, while it was a little worse in the along-track direction. The mean values were $19.9,174.6$ and $81.0 \mathrm{~cm}$ in three directions. The accuracies of the GEO satellites were worse than for IGSO and MEO satellites, especially in the along-track direction. The mean values are $47.3,454.5$ and $37.8 \mathrm{~cm}$ respectively.

In addition, we also analyzed the discontinuities between updates. For each orbit file, the satellite positions were calculated based on the interval from 1 to 4 hours of the predicted half and were compared to those calculated from the next ephemeris file. The mean RMS for all satellite systems and types between each update were plotted in Fig. 4, and the actual mean values during the period are given in Table 3. Similar to the results previously, the discontinuities for GPS are most stable and smallest with a mean RMS of 1.3, 8.1, and $6.4 \mathrm{~cm}$ in the radial, along-track and cross-track directions, respectively. Such discontinuities can be mostly absorbed in RT clock offset estimation. Compared with that, the discontinuities of BeiDou satellites were much bigger, especially those of the GEO satellites, of which the mean RMS in the radial direction reached up to $82.1 \mathrm{~cm}$. The RMS in the along-track direction varied much more, and reached up to nearly 10 meters. The mean RMS of the IGSO satellites was $33.4,129.0$ and $98.7 \mathrm{~cm}$ each, and the accuracies of MEO satellites were similar, with average RMS of 25.7, 197.6 and $70.6 \mathrm{~cm}$ each. Thus, we applied the previous smoothing method to eliminate such big discontinuities in clock offset estimation.

Selecting the final GNSS clock offsets from IGG, we also evaluated the accuracy of RT clock offsets. Before the comparison, the impact of the differences of satellite orbit fixed in calculating the satellite clock offsets is corrected by the method proposed by Kouba and Springer (2011) and can be expressed as follows:

$$
\Delta c l k_{S A T}=\left(X_{R T}-X_{I G G}\right) \square X_{R T} / R_{S A T} / c
$$

$X_{R T}$ and $X_{I G G}$ represent the satellite position calculated from the ephemeris fixed in

generating the two types of satellite clock offsets, $R_{S A T}$ denotes the satellite radius vector.

The accuracy results of all satellites were plotted in Fig. 5. We can find that the STDs of all GPS satellites were smaller than $0.1 \mathrm{~ns}$ and the average value was $0.07 \mathrm{~ns}$. The STDs of the BeiDou satellites were bigger than for GPS, but were still smaller than $0.4 \mathrm{~ns}$ except for C12. The mean value was $0.25 \mathrm{~ns}$. Considering the accuracy of BeiDou satellite orbits, we can see that most of the orbit errors can be absorbed by the satellite clock offsets, which provided a good possibility in realizing highly precise user positioning based on BeiDou observations. Different from STD, the RMS was much bigger, of which the average values were 1.27 and $2.37 \mathrm{~ns}$ for GPS and BeiDou, respectively. However, the impact of such big RMS on the user positioning accuracy is small because this bias will be absorbed by the ambiguities of phase observations in user positioning.

\subsection{Performance of RT kinematic PPP}

Fig. 6 plots the number of satellites tracked, PDOP value and the positioning errors of WILU on February 14, 2017. We can find from the figure that the number of GPS and BeiDou satellites tracked was comparable. The mean number of GPS satellites was 9, while it was 10 for BeiDou due to the special configuration of the constellation and the station position. Different from that, 
the PDOP value of the GPS-only solution was smaller than that of the BeiDou-only solution at most of the time. Especially, the PDOP value of the BeiDou-only solution varied greatly and became bigger than 10 due to the missing of several satellites. This can also be attributed to the current constellation of BeiDou, of which most of the satellites were GEO and IGSO. When combining the observations of the two GNSS, the number of satellites tracked was always bigger than 15 except for two epochs, and reached up to 19 on average. Corresponding, the PDOP value became smaller and more stable, which suggests the possibility in improving the performance.

As revealed by the positioning results, an initialization time was required in all three solutions. After that, accurate positioning results were achieved with an accuracy of 20 and $40 \mathrm{~cm}$ in the horizontal and vertical directions, respectively. Among the solutions, the differences between the GPS-only and GPS/BeiDou solution were small. Compared with that, the positioning errors of the BeiDou-only solution were much bigger. During some periods, the positioning errors of the Up component were bigger than $20 \mathrm{~cm}$.

Following on, we analyzed the initialization time and positioning accuracy in detail. In order to quantify the initialization time required in all solutions, the observations were split every 2 hours and reprocessed. Then, the mean RMS of the positioning errors as a function of the time since RT kinematic PPP started were summarized and plotted in Fig. 7. In addition, the actual values after 15 and 30 minutes each are given in Table 4. Comparing the initialization process of the two single system solutions, the GPS-only solution converged faster than the BeiDou-only solution in all three directions. The RMS in the three directions after 15 minutes is $9.2,23.0$ and $24.4 \mathrm{~cm}$ each in the GPS-only solution, while $17.8,40.4$ and $78.7 \mathrm{~cm}$ each in the BeiDou-only solution. After 30 minutes, the RMS improved to $5.1,15.3$ and $15.5 \mathrm{~cm}$ each in the GPS-only solution, while to only $12.2,30.0$ and $45.6 \mathrm{~cm}$ each in the BeiDou-only solution. This relatively slower convergence speed of the BeiDou-only solution is correlated with the lower accuracy of the satellite orbit/clock results and the slower varying satellite geometry.

In the GPS/BeiDou solution, the convergence speed was faster than for the two single-system solutions, especially in the Up component. Compared with the GPS-only solution, the positioning accuracies after 15 minutes were 37.6, 26.1 and 36.0 percent improved. This improvement after 30 minutes became 43.7, 36.9 and 45.0 percent in three directions. These results reveal that for time-critical applications observations from multiple GNSS are beneficial.

Next, the observations were processed continuously every day to evaluate the positioning accuracy of all solutions. When summarizing the accuracy, the positioning results of the first two hours in each day were ignored because of the initialization process. The positioning accuracy of each station and solution on February 14, 2017 are plotted in Fig. 8. We can find that decimeter accuracy positioning results are achieved in the BeiDou-only solution and the accuracies were comparable between different stations, except for GGTN. These revealed that the system based on BeiDou-only observations was capable of providing decimeter accuracy positioning service for all users in the region despite the relatively lower orbit accuracy. The mean accuracy of the North, East and Up component was 4.9, 6.6, and $22.7 \mathrm{~cm}$, respectively. Compared with the BeiDou-only solution, the GPS-only solution was more accurate. The RMS was smaller than 5 and $10 \mathrm{~cm}$ each in the horizontal and vertical direction for all stations. However, different from the initialization time, the improvement in the positioning accuracy was much lower when adding the BeiDou observations to those from GPS. The differences in the accuracy between the two solutions were within $2 \mathrm{~cm}$. 
Process the observations of four days, and calculate the daily average accuracy of the three solutions. The results are plotted in Fig. 9. The accuracies between different days for all solutions were similar, which validates the stability of the established positioning system. For the BeiDou-only solution, the mean accuracies of three components were $5.2,8.1$ and $17.8 \mathrm{~cm}$ for the North, East and Up components, respectively. For the GPS-only solution, the accuracies were better than $6 \mathrm{~cm}$ on all days, and the mean accuracies of three components were 1.5, 3.0, and $4.7 \mathrm{~cm}$. When combining the observations, about 20.9\% improvement of the East component was achieved, but no obvious improvement can be seen in the North and Up directions. Further improvement is expected to be achieved with increasing accuracy of BeiDou-only PPP.

\section{Conclusions and outlook}

In this work, a regional RT precise positioning system based on GPS/BeiDou observations was established and the performance of the system was analyzed in detail. Three hourly updated satellite orbits were imported and then RT satellite clock offsets were estimated by processing RT observation/navigation data streams to generate the correction messages. At the user side, ionosphere free observations from GPS/BeiDou were processed to realize high accuracy user positioning and an adaptive weighting scheme based on Helmert variance component estimation was applied.

The average accuracy of the satellite orbit applied in the system reached $2.8,7.7$ and $5.5 \mathrm{~cm}$ in the radial, along-track and cross-track direction for GPS, while $33.8,267.5$, and $79.5 \mathrm{~cm}$ for BeiDou. Based on the orbit, the STDs of the RT clock offsets were 0.07 and 0.25 ns for GPS and BeiDou respectively. In terms of user positioning, the GPS-only solution converged much faster than the BeiDou-only solution. The accuracy of the North, East and Up components reached 5.1, 15.3 and $15.5 \mathrm{~cm}$ each in the GPS-only solution after 30 minutes. However, it only reached 12.2, 30.0 and $45.6 \mathrm{~cm}$ for the BeiDou-only solution at the same time. When combining the observations, the solution converged much faster than the two single-system solutions. The accuracies reached 5.7, 17.0 and $15.6 \mathrm{~cm}$ after only 15 minutes. Thus, it would be effective in time-critical applications. After the initialization process, an accuracy of 5.2, 8.1 and $17.8 \mathrm{~cm}$ in the North, East and Up directions was achieved in the BeiDou-only solution while the performance was comparable for all user stations. These results validate that the regional RT system is capable of providing decimeter positioning services for users based on BeiDou observations. The average accuracies of the three components in the GPS-only solution were 1.5, 3.0, and $4.7 \mathrm{~cm}$ each. However, only about $20.9 \%$ improvement in the East direction was observed when adding BeiDou observations on top of GPS.

In future, we will focus on improving the accuracy and stability of the regional RT system, especially the accuracy of BeiDou satellite orbit determination and RT clock offset estimation. In addition, the incorporation of GLONASS and Galileo observations to further improve the performance of the service will also be addressed.

\section{Acknowledgments}

The work is funded by the National Natural Science Foundation of China (Grant No. 41404017, 41574015, 41621091), the National Key Research Program of China "Collaborative Precision 
Positioning Project" (No. 2016YFB0501900), the Fonds National de la Recherche, Luxembourg (Reference No. 6823109) and supported by iGMAS. We thank AUSCORS in providing the real-time stream. The IGS and MGEX are also high appreciated in providing GNSS data.

\section{References}

Böhm, J., Niell, A., Tregoning, P., Schuh, H., 2006. Global Mapping Function (GMF): A new empirical mapping function based on numerical weather model data. Geophys. Res. Lett., 33, L07304, doi:10.1029/2005GL025546

Cai, C., Gao, Y., 2013. Modeling and assessment of combined GPS/GLONASS precise point positioning. GPS Solut., 17(2), 223-236

Cai, C., Pan, L., Gao, Y., 2014. A precise weighting approach with application to combined L1/B1 GPS/BeiDou positioning. J. Navig., 67(5), 911-925

Caissy, M., Agrotis, L., Weber, G., et al., 2012. The International GNSS Real-Time Service. GPS World, 23(6), 52-58

Dach, R., Brockman, E., Schaer, S., et al., 2009. GNSS processing at CODE: status report. J Geod. 83(3-4), 353-365

de Haan, S., Holleman, I., Holtslag, A.A.M., 2009. Real-time water vapor maps from a GPS surface network: Construction, validation, and applications. J. Appl. Meteorol. Climatol., 48(7), $1302-1316$

Defraigne, P., Aerts, W., Pottiaux, E., 2015. Monitoring of UTC (k)'s using PPP and IGS real-time products. GPS Solut., 19(1), 165-172

Douša, J., 2010. The impact of errors in predicted GPS orbits on zenith troposphere delay estimation. GPS Solut., 14(3), 229-239

Dow, J.M., Neilan, R.E., Rizos, C., 2009. The international GNSS service in a changing landscape of global navigation satellite systems. J Geod., 83(3-4), 191-198

Elsobeiey, M., Al-Harbi, S., 2016. Performance of real-time Precise Point Positioning using IGS real-time service. GPS Solut., 20(3), 565-571

Ge, M., Chen, J., Douša, J., et al., 2012. A computationally efficient approach for estimating high-rate satellite clock corrections in realtime. GPS Solut., 16(1), 9-17

Geng, J., Teferle, F.N., Meng, X., et al., 2011. Towards PPP-RTK: Ambiguity resolution in real-time precise point positioning. Adv. Space Res., 47(10), 1664-1673 
Guo, J., Xu, X., Zhao, Q., et al., 2016. Precise orbit determination for quad-constellation satellites at Wuhan University: Strategy, result validation, and comparison. J. Geod., 90(2), 143-159

Hadas, T., Bosy, J., 2015. IGS RTS precise orbits and clocks verification and quality degradation over time. GPS Solut., 19(1), 93-105

Janssen, V., 2009. Precision Rules! How to establish an AusCORS site. Position, (44), 64-66

Kouba, J., Springer, T., 2001. New IGS station and satellite clock combination. GPS Solut., 4(4), 31-36

Laurichesse, D., 2015. IGSMAIL-7183 New CNES real time products including BeiDou. https://igscb.jpl.nasa.gov/pipermail/igsmail/2015/008373.html

Leandro, R., Landau, H., Nitschke, M., et al., 2011. RTX positioning: the next generation of cm-accurate real-time GNSS positioning. In: Proc. ION GNSS 2011, Portland. OR. September 2011, pp. $1460-1475$

Li, M., Qu, L., Zhao, Q., et al., 2014. Precise point positioning with the BeiDou navigation satellite system. Sensors, 14(1), 927-943

Li, X., Ge, M., Dai, X., et al., 2015. Accuracy and reliability of multi-GNSS real-time precise positioning: GPS, GLONASS, BeiDou, and Galileo. J. Geod., 89(6), 607-635

Lou, Y., Zhang, W., Wang, C., et al., 2014. The impact of orbital errors on the estimation of satellite clock errors and PPP. Adv. Space Res., 54(8), 1571-1580

Montenbruck, O., Hauschild, A., Steigenberger, P., et al., 2013. Initial assessment of the COMPASS/BeiDou-2 regional navigation satellite system. GPS Solut., 17(2), 211-222

Montenbruck, O., Steigenberger, P., Prange, L., et al., 2017. The Multi-GNSS Experiment (MGEX) of the International GNSS Service (IGS) - Achievements, prospects and challenges. Adv. Space Res., 59(7), 1671-1697

Saastamoinen, J., 1972. Atmospheric correction for the troposphere and stratosphere in radio ranging of satellites in the user of artificial satellites for geodesy. Geophys. Monogr. Ser., vol. 15, American Geophysical Union, Washington, DC, 247-251

Steigenberger, P., Hugentobler, U., Hauschild, A., et al., 2013. Orbit and clock analysis of Compass GEO and IGSO satellites. J. Geod., 87(6), 515-525

Sun, F.P., Liu, S., Zhu, X.H., et al. 2012. Research and progress of Beidou satellite navigation system. Sci. China Inf. Sci., 55(12), 2899-2907 
Tegedor, J., de Jong, K., Liu, X., et al., 2015. Real-Time Precise Point Positioning Using BeiDou. IAG Symp. vol 143, 665-671

Tegedor, J., Øvstedal, O., Vigen, E., 2014. Precise orbit determination and point positioning using GPS, Glonass, Galileo and BeiDou. J. Geod. Sci., 4(1), 65-73

Tegedor, J., Lapucha, D., Øvstedal, O., et al., 2015. The New G4 Service: Multi-constellation Precise Point Positioning Including GPS, GLONASS, Galileo and BeiDou, In: Proc. ION GNSS+ 2015, Tampa, Florida. September 2015, pp. 1089-1095

Tiberius, C.C.J.M., 1998. Recursive data processing for kinematic GPS surveying. Ph.D. Thesis, Department of Mathematical Geodesy and Positioning, Delft University of Technology

Wanninger, L., Beer, S., 2015. BeiDou satellite-induced code pseudorange variations: diagnosis and therapy. GPS Solut., 19(4), 639-648

Wu, J.T., Wu, S.C., Hajj, G.A., et al., 1993. Effects of antenna orientation on GPS carrier phase. Manuscr. Geod., 18, 91-98

Yuan Y, Tan B, Ding W, et al., 2016. Status and Progresses at IGG GNSS data Analysis Center. http://acc.igs.org/workshop2016/posters/Yunbin_Yuan_Status_and_Progress_IGG.pdf

Zhang, W., Lou, Y., Gu, S., et al., 2016. Joint estimation of GPS/BDS real-time clocks and initial results. GPS Solut., 20(4), 665-672

Zumberge, J.F., Heflin, M.B., Jefferson, D.C., et al., 1997. Precise point positioning for the efficient and robust analysis of GPS data from large networks. J. Geophys. Res., 102(B3), 5005-5017 
Table 1 Settings in RT clock offset estimation and kinematic PPP

\begin{tabular}{|c|c|}
\hline Options & Settings \\
\hline $\begin{array}{l}\text { Observation } \\
\text { weighting }\end{array}$ & $\begin{array}{l}\text { Elevation dependent weighting; } 0.006 \mathrm{~m}, 0.6 \mathrm{~m} \text { for one way phase and code observation of } \\
\text { BeiDou GEO satellites in the zenith direction, } 0.003 \mathrm{~m}, 0.3 \mathrm{~m} \text { otherwise }\end{array}$ \\
\hline Sampling interval & 3s for RT clock offset estimation, $5 \mathrm{~s}$ for RT PPP \\
\hline $\begin{array}{l}\text { Elevation cut-off } \\
\text { angle }\end{array}$ & $7^{\circ}$ \\
\hline Troposphere & $\begin{array}{l}\text { Hydrostatic: Saastamoinen model (Saastamoinen, 1972) } \\
\text { Non-hydrostatic: Random walk process, and the noise intensity is } 0.05 \mathrm{~mm} / \sqrt{\mathrm{s}} \\
\text { Mapping function: GMF (Böhm et al., 2006) }\end{array}$ \\
\hline Ambiguity & $\begin{array}{l}\text { Clock offset estimation: Eliminated by epoch differencing } \\
\text { PPP: Estimated as constant float values }\end{array}$ \\
\hline Phase wind-up & Corrected (Wu et al., 1993) \\
\hline
\end{tabular}




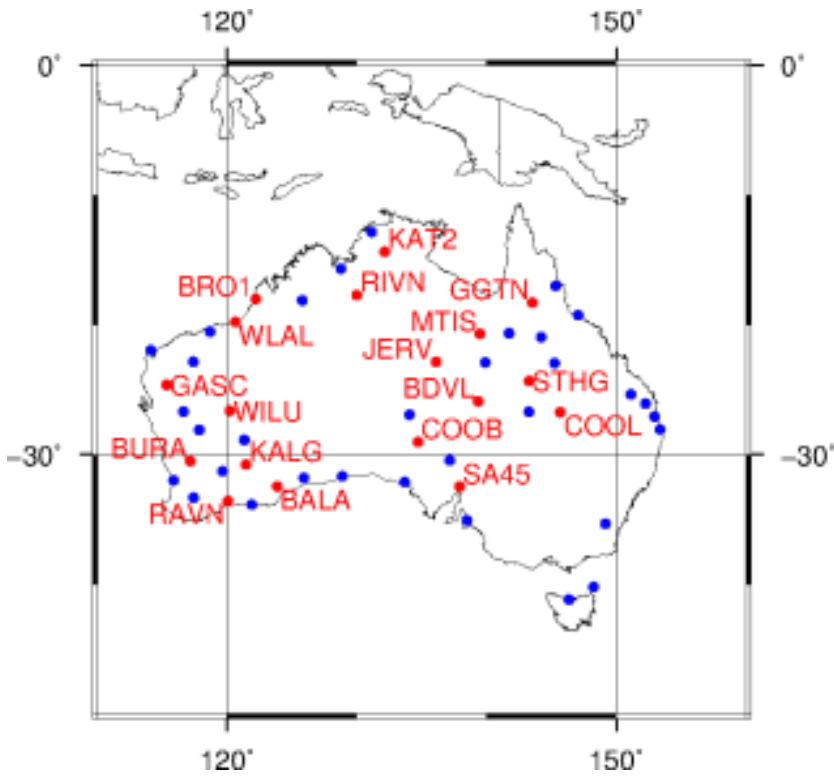

Fig. 1 Distribution of reference and user stations selected in the analysis

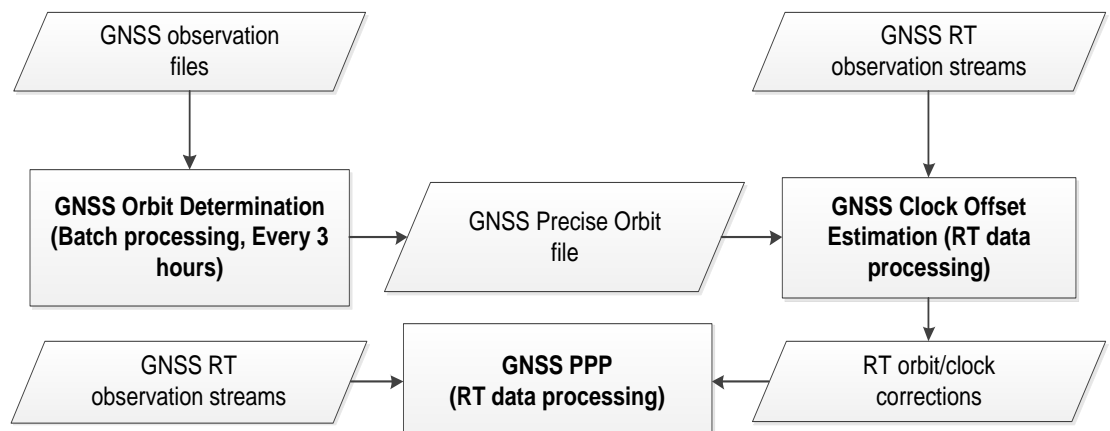

Fig. 2 Structure of RT precise positioning system 

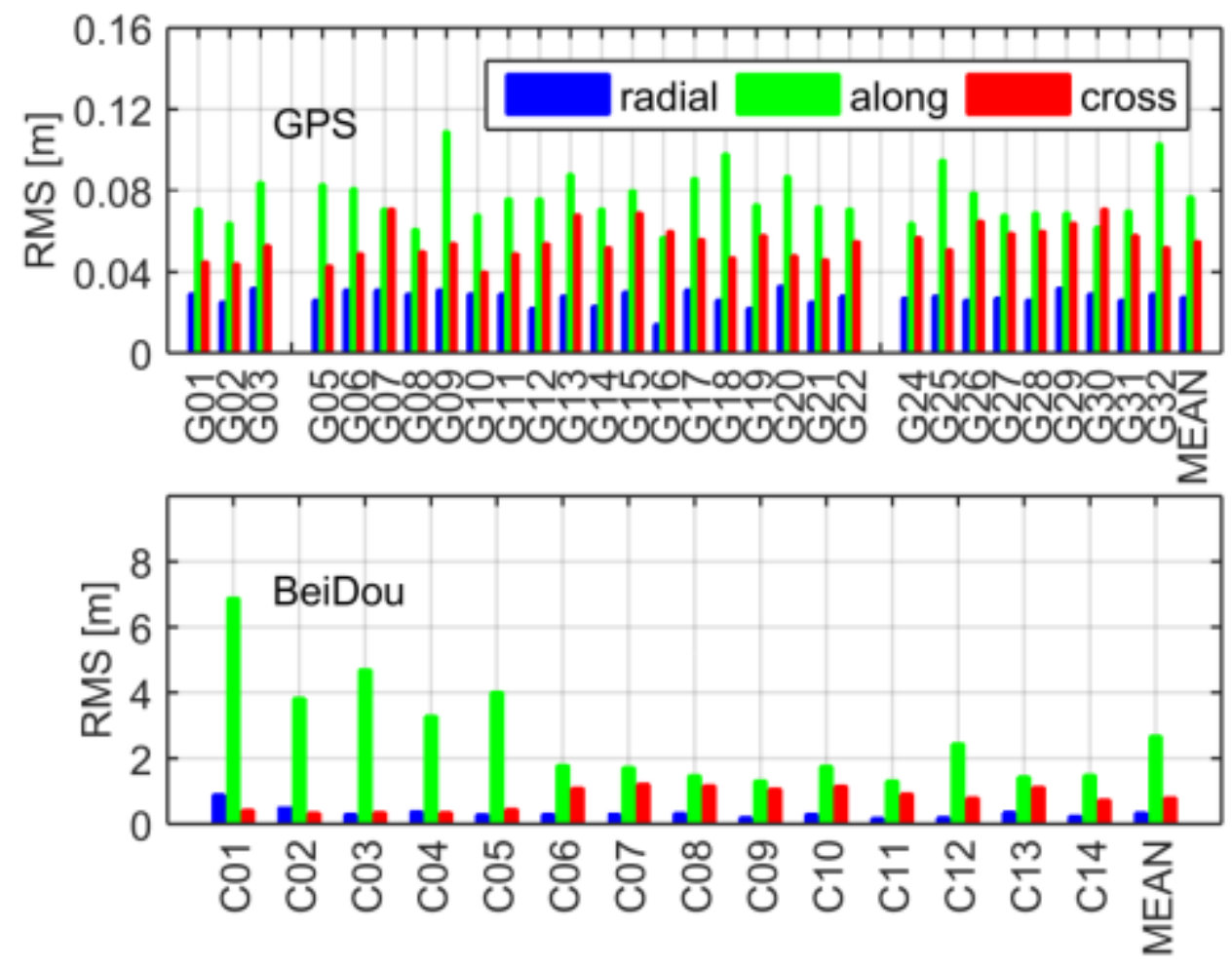

Fig. 3 Accuracy of satellite orbits comparing with the final GNSS orbits from IGG

Table 2 Average RMS values of satellite orbits comparing with the final GNSS orbits from IGG

\begin{tabular}{cccc}
\hline Satellite & Radial $(\mathrm{cm})$ & Along $(\mathrm{cm})$ & Cross $(\mathrm{cm})$ \\
\hline GPS & 2.8 & 7.7 & 5.5 \\
\hline BeiDou GEO & 47.3 & 454.5 & 37.8 \\
\hline BeiDou IGSO & 29.5 & 158.1 & 113.5 \\
\hline BeiDou MEO & 19.9 & 174.6 & 81.0 \\
\hline
\end{tabular}

Table 3 Average RMS values of the discontinuities between satellite orbit updates

\begin{tabular}{cccc} 
Satellite & Radial $(\mathrm{cm})$ & Along $(\mathrm{cm})$ & Cross $(\mathrm{cm})$ \\
\hline GPS & 1.3 & 8.1 & 6.4 \\
\hline BeiDou GEO & 82.1 & 267.4 & 36.9 \\
\hline BeiDou IGSO & 33.4 & 129.0 & 98.7 \\
\hline BeiDou MEO & 25.7 & 197.6 & 70.6
\end{tabular}



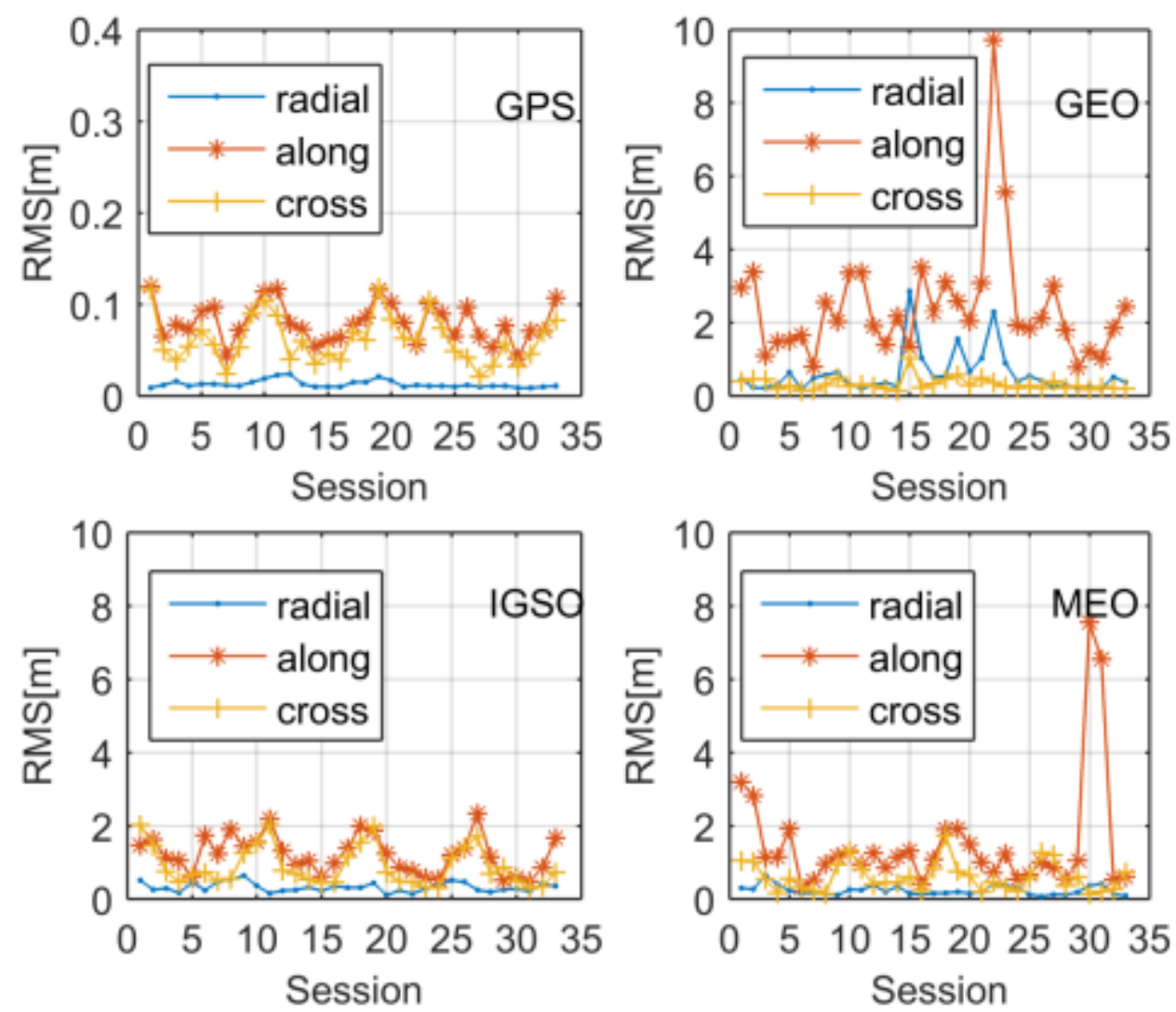

Fig. 4 Discontinuities of satellite orbits between sessions
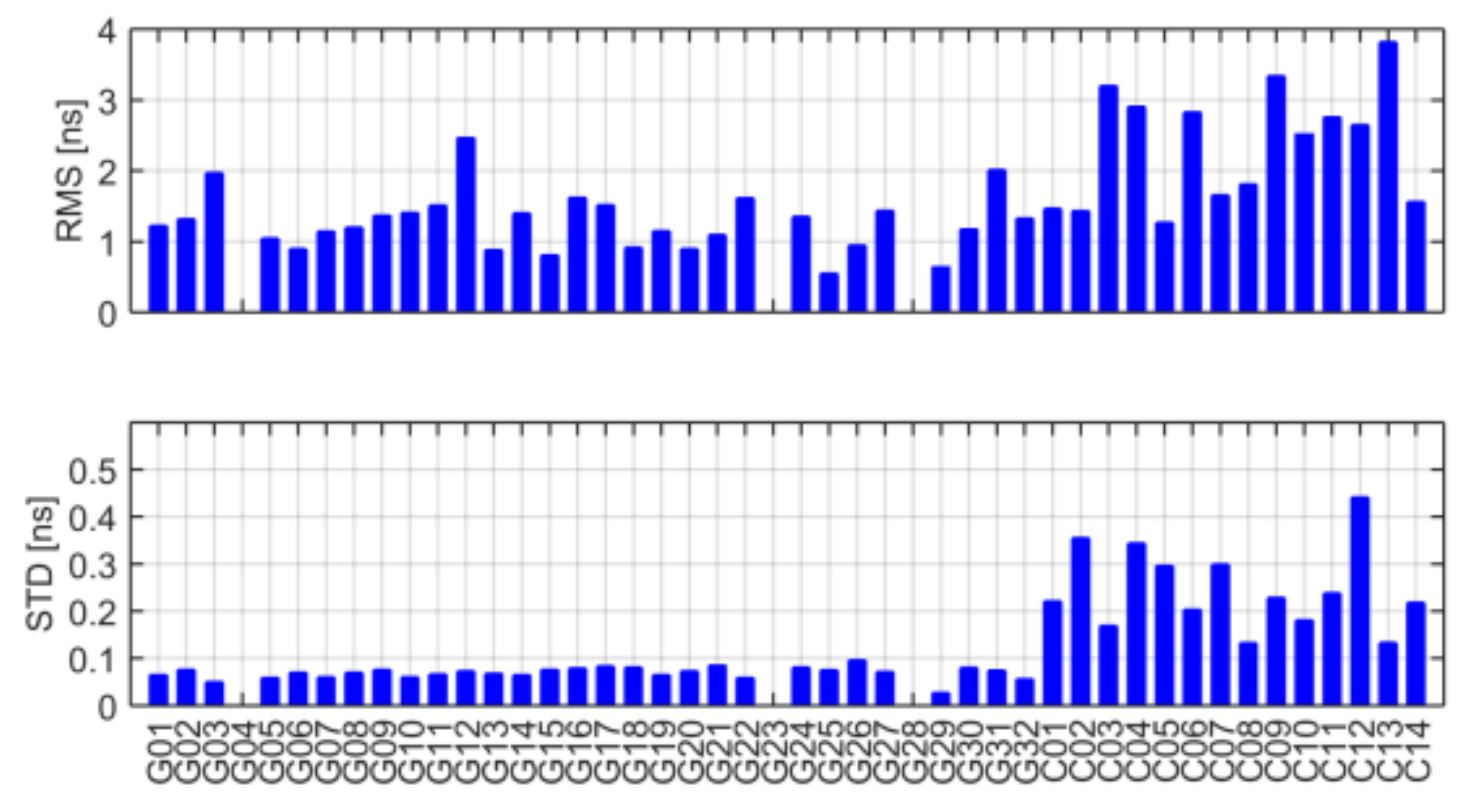

Fig. 5 Accuracy of RT clock offsets with respect to the final clock results from IGG 

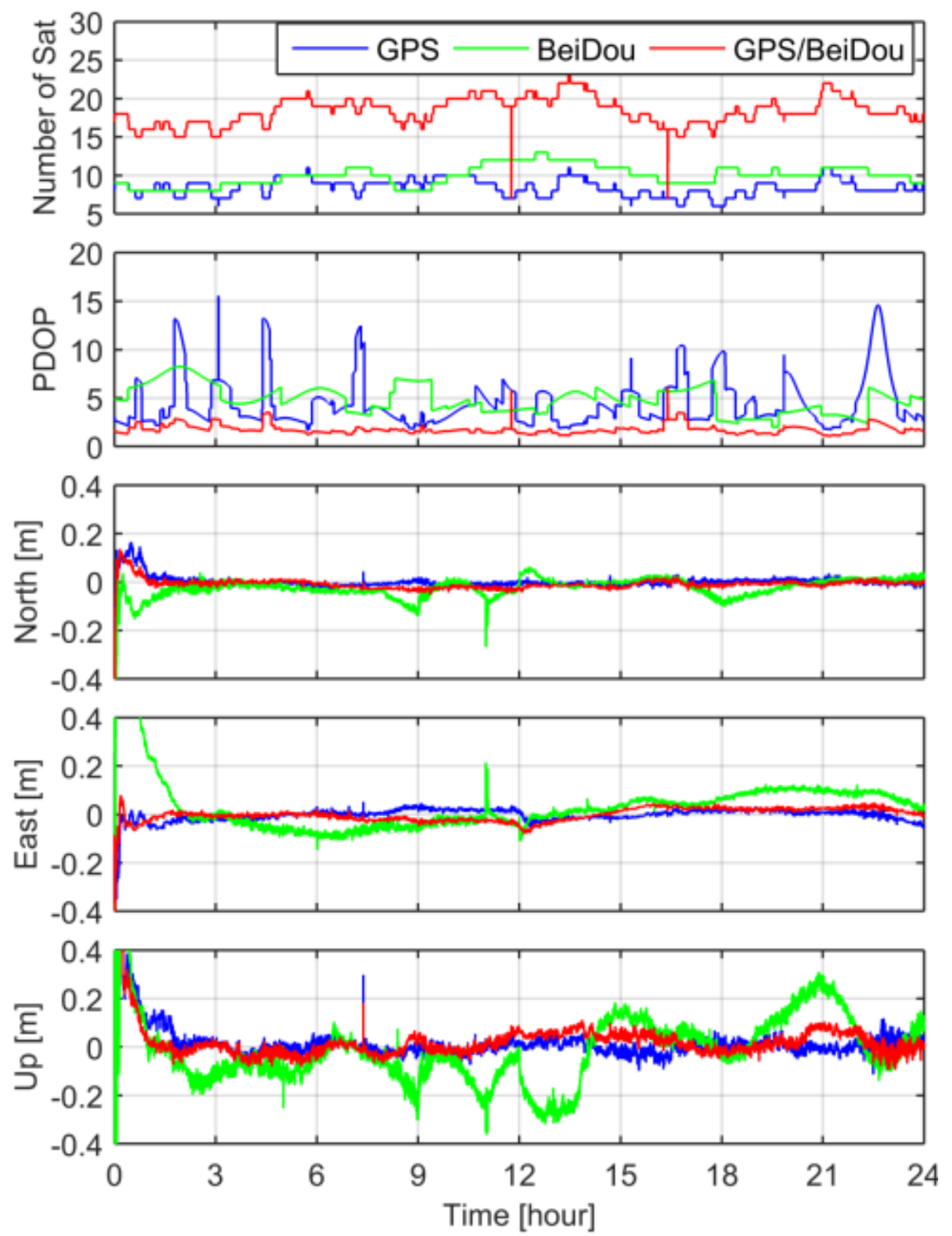

Fig. 6 Number of satellites, PDOP value, and positioning errors of station WILU on February 14, 2017 

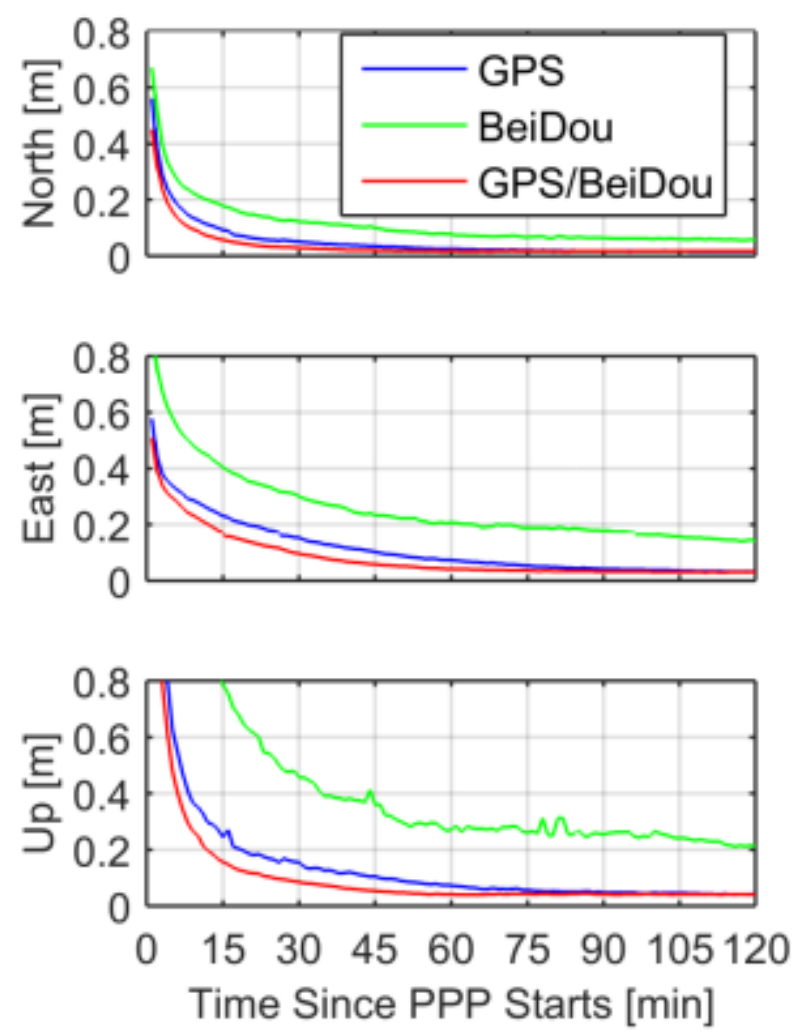

Fig. 7 Mean RMS as a function of the time since RT kinematic PPP started Table 4 Mean RMS of RT kinematic PPP after an initialization process of 15 and 30 minutes

\begin{tabular}{ccccccc}
\hline \multirow{2}{*}{ Solutions } & \multicolumn{3}{c}{$15 \mathrm{~min}$} & \multicolumn{3}{c}{$30 \mathrm{~min}$} \\
\cline { 2 - 7 } & North $(\mathrm{cm})$ & East $(\mathrm{cm})$ & Up $(\mathrm{cm})$ & North $(\mathrm{cm})$ & East $(\mathrm{cm})$ & Up $(\mathrm{cm})$ \\
\hline GPS & 9.2 & 23.0 & 24.4 & 5.1 & 15.3 & 15.5 \\
\hline BeiDou & 17.8 & 40.4 & 78.7 & 12.2 & 30.0 & 45.6 \\
\hline GPS/BeiDou & 5.7 & 17.0 & 15.6 & 2.8 & 9.7 & 8.5
\end{tabular}



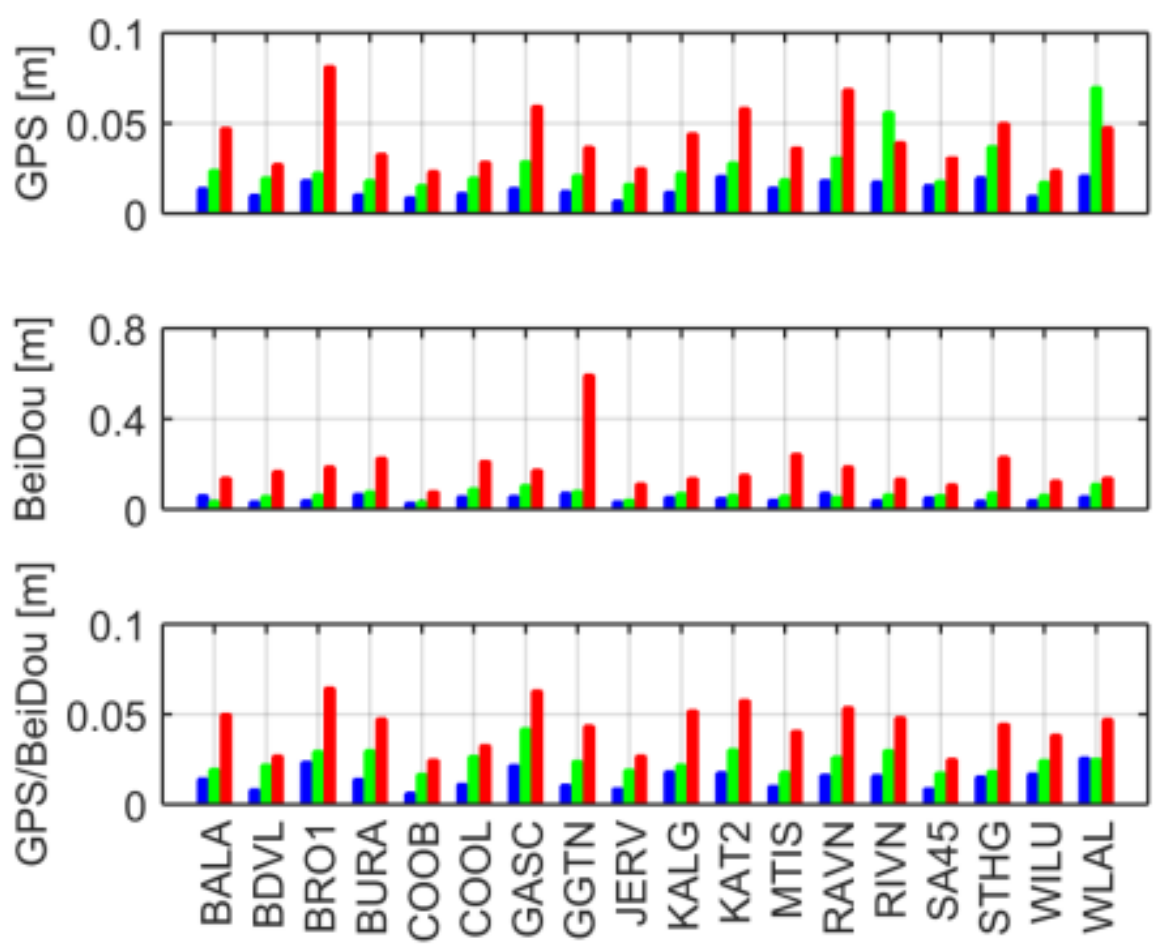

Fig. 8 Positioning accuracy of all stations and solutions on February 14, 2017
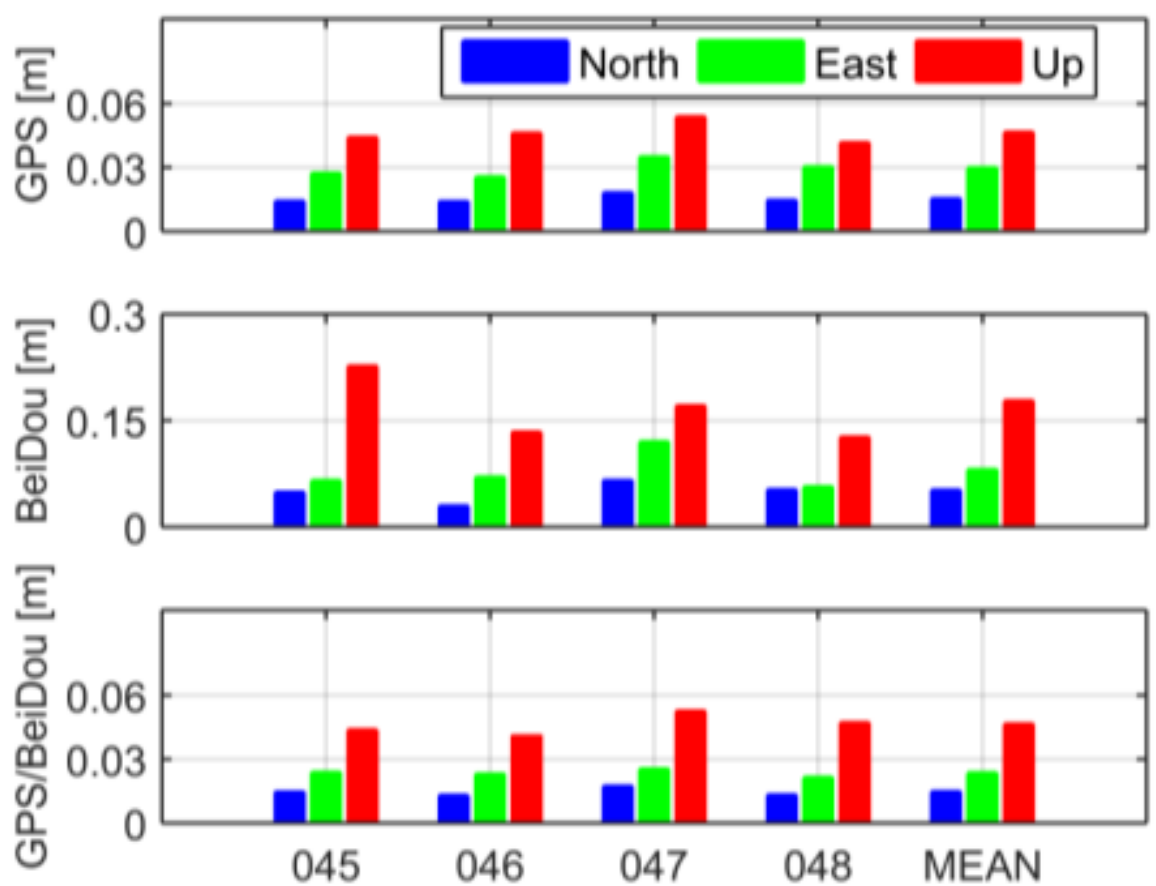

Fig. 9 Daily average positioning accuracy of different solutions

Table 5 Average RMS values of positioning accuracy in different solutions

\begin{tabular}{cccc}
\hline Systems & North $(\mathrm{cm})$ & East $(\mathrm{cm})$ & Up $(\mathrm{cm})$ \\
\hline GPS & 1.5 & 3.0 & 4.7 \\
\hline BeiDou & 5.2 & 8.1 & 17.8 \\
\hline GPS/BeiDou & 1.5 & 2.4 & 4.7 \\
\hline
\end{tabular}

